La incorporación de las humanidades y ciencias socio-conductuales en la educación médica: ¿cuál es el problema y qué se debe hacer?

Roger Ruiz Moral

\title{
LA INCORPORACIÓN DE LAS HUMANIDADES Y CIENCIAS SOCIO- CONDUCTUALES EN LA EDUCACIÓN MÉDICA: ¿CUÁL ES EL PROBLEMA Y QUÉ SE DEBE HACER?
}

\section{Roger Ruiz Moral}

\begin{abstract}
Resumen: Este artículo describe las dificultades que las humanidades y ciencias sociales y de la conducta (H\&CSC) tradicionalmente tienen para incorporarse como miembros de pleno derecho en la educación médica y analiza las razones por las que esto ocurre desde inicios del siglo XX. Se identifica la hegemonía en la educación médica de la biomedicina como el núcleo central de esta dificultad y consecuencia de las múltiples barreras y problemas descritos para esta incorporación. Utilizando la metáfora de las "muñecas rusas" y los conceptos de "dominio y campo epistémicos", se ofrecen argumentos que permiten comprender el alcance y consecuencias de esta "hegemonía ideológica". Finalmente se proponen dos estrategias que en la medida en la que responden a los argumentos esenciales en los que se basa el predominio biomédico pueden considerarse de suficiente trascendencia y alcance para contrapesar la hegemonía biomédica y contribuir a una integración efectiva de las H\&CSC: por un aparte la necesidad de incorporar como eje educativo una "razón ampliada" que incorpore perspectivas epistemológicas y antropológicas que superen al empirismo materialista reivindicando la validez de metodologías diferentes a las meramente cuantitativas experimentales y por otra insistir también en la utilidad práctica de las H\&CSC y sus metodologías para resolver los problemas actuales que plantea la clínica.
\end{abstract}

Palabras clave: humanidades/ ciencias sociales y conductuales/ biomedicina/ educación médica/ razón abierta/ epistemología/ ideología.

\section{Abstract:}

This article describes the difficulties that the humanities and social and behavioral sciences (H\&CSC) traditionally have for incorporating themselves as full members in medical education analysing the reasons why this came about since the beginning of the 20th century. The hegemony in medical education of biomedicine is identified as the core of this difficulty and consequence of the multiple barriers and problems described for this incorporation. Using the metaphor of the "russian dolls" and the concepts of "domain and epistemic field", arguments for understanding the scope and consequences of this "ideological hegemony" are offered. Finally, two strategies are also proposed which, to the extent they respond to the essential arguments on which biomedical hegemony is founded, they can be considered of enough importance and scope to counterweight the biomedical hegemony and contribute to an effective integration of H\&CSC: the need to incorporate as educational axis an "expanded ratio" that incorporates epistemological and anthropological perspectives that overcome materialistic empiricism, claiming the validity of methodologies different from the purely quantitative ones, and second, to insist on the practical utility of $\mathrm{H} \& \mathrm{CSC}$ and their methodologies for solving the current problems the medical practice need to face.

Keywords: humanities/ social and behavioral sciences/ biomedicine/ medical education/ expanded reason/ epistemology/ ideology.

Artículo recibido: 21 noviembre 2018; aceptado: 8 marzo 2019. 
La incorporación de las humanidades y ciencias socio-conductuales en la educación médica: ¿cuál es el problema y qué se debe hacer?

Roger Ruiz Moral

\section{INTRODUCCIÓN}

Desde principios del siglo $X X$ se ha venido reconocido que la ciencia biomédica representa el conocimiento básico para la formación de los estudiantes de medicina $(1,2)$. Sin embargo, a la vez a lo largo del último siglo y lo que llevamos del siglo XXI, se ha insistido repetidamente en la necesidad de proponer cambios sobre la forma en la que estos estudiantes debían de ser seleccionados y educados, ya que se reconoce también que la ciencia biomédica es insuficiente para formar a un profesional de la salud competente $(3,4)$. Los cambios que de forma insistente se han propuesto se dirigen a: limitar la super-especialización e introducir el generalismo y comunitarismo; promover un proceso de selección de los estudiantes de medicina que haga énfasis en un estudiante con capacidad de pensar y no meramente de memorizar; realizar una revisión crítica de lo que aporta el conocimiento biomédico clínico; afrontar el problema de la sobrecarga curricular en medicina y plantear una mayor y más relevante presencia de las humanidades y las ciencias sociales en los curricula de ciencias de la salud. En este artículo me centraré en revisar algunos aspectos de por qué la medicina no puede ser ni practicada ni enseñada bajo una perspectiva exclusivamente basada en la biomedicina y la tecnología. Y la necesidad de incorporar las humanidades y las ciencias sociales y conductuales (H\&CSC) en la educación médica. Con éste propósito, expondré algunos de los avatares de esta reivindicación, presentando argumentos que apoyan las razones por las que la misma no tiene éxito para finalmente apuntar dos posibles estrategias que a mi juicio podrían contribuir a una más exitosa incorporación de estas H\&CSC en la educación médica.

A lo largo de todos estos años las advertencias de este tipo han sido constantes en el ámbito de la educación médica, apelando una y otra vez a esa necesidad cada vez más perentoria de incluir reformas curriculares consistentes en el sentido antes apuntado $(3,5,6)$. La realidad es que las reformas curriculares que se han ido proponiendo y que en muchos casos se han llevado en mayor o menor medida a la práctica, sin embargo, no son consideradas suficientes tanto en cantidad como en forma. Estas reformas no acaban de dar respuesta a los objetivos prácticos que se pretenden con la incorporación de las H\&CSC. En 1975 una encuesta 
La incorporación de las humanidades y ciencias socio-conductuales en la educación médica: ¿cuál es el problema y qué se debe hacer?

Roger Ruiz Moral

realizada a los decanos de las escuelas de medicina americanas encontró que "casi el 90\%" de ellos apoyaban la inclusión y un mayor peso en el currículum de las ciencias sociales, conductuales y las humanidades. Sin embargo, a la vez estos decanos veían la posibilidad de realizar este cambio de una forma marginal o escasa; ellos mismos se consideraban con poca capacidad para influenciar en esta dirección (7).

El sociólogo S Bloom (8) llamó la atención sobre la paradoja de que a pesar de que la práctica clínica ha cambiado en los últimos años enormemente, sin embargo, la educación médica no lo ha hecho. Para este autor, la razón de que esto sea así es que la educación médica está al servicio de la biomedicina y de sus avances científico-técnicos, y no realmente al servicio de las necesidades siempre cambiantes de la práctica clínica. Argumenta que la educación médica ha integrado con éxito durante todos estos años el conocimiento biomédico y sus avances. Bloom destaca el relevante papel que en este proceso juega la investigación biomédica y argumenta que "la misión científica de la medicina académica ha desplazado su responsabilidad social de entrenar para afrontar las necesidades de atención sanitaria más básicas de la sociedad", “..la manifiesta misión humanista de la educación médica es poco más que una fachada para la misión de la investigación (biomédica) que sería la preocupación real de las instituciones educativas" (8). Por otra parte, Christakis (9) analizó también el contenido de los informes educativos médicos y, al igual que otros autores (10), señaló la aparente similitud de las propuestas de cambio que se suceden de forma periódica en la educación médica. Según este autor, en realidad no existirían unas propuestas de reformas reales para abordar e incorporar de manera significativa las necesidades sociales y, a diferencia de Bloom, sostiene que lo que estas reformas pretenden no es tanto el reforzar la investigación biomédica como el reforzar la profesión médica (sus valores y sus modos de autorregulación), pero una vez más no responderían a la necesidad de adaptar la enseñanza a la realidad de la clínica.

\section{LA FUERZA DE LA IDEOLOGÍA COMO BARRERA DEL CAMBIO}

En los últimos años se han sucedido los estudios que han explorado las barreras para la integración de las ciencias sociales y conductuales en el currículum 
La incorporación de las humanidades y ciencias socio-conductuales en la educación médica: ¿cuál es el problema y qué se debe hacer?

Roger Ruiz Moral

general de medicina. La mayoría de estos estudios reconocen que, a pesar de la necesidad de su incorporación y de que su presencia se ha hecho efectiva en un gran número de escuelas de medicina, ésta presencia sin embargo (como he señalado antes) presenta problemas en casi todos los lugares donde se ha intentado. Las razones que están detrás de este fracaso pueden ser las apuntadas por Bloom (8) o Christakis (9), o tal vez otras (más adelante ofreceré otra razón ciertamente complementaria con las ya expuestas), pero la realidad es que en el fondo existe la percepción entre educadores y estudiantes o residentes de que tales materias en las facultades de medicina son algo que "está bien conocer" (nice to know) pero no son consideradas como algo que "sea preciso conocer" (need to know) (11). Esta percepción nice to know revela la consideración de un status de las H\&CC secundario o supeditado al status que tienen las ciencias biomédicas al que antes nos referíamos como ideología preponderante en la educación médica.

La mayoría de los estudios que exploran los problemas de implementación de las H\&CC en las escuelas de medicina coinciden en señalar la existencia de una serie de barreras. Siguiendo la revisión de Tabatabaei et al (2016) (12), estas barreras pueden agruparse en 7 dominios y 23 componentes repartidos en esos dominios: liderazgo ineficaz, problemas relacionados con el profesorado que imparte estas materias, con la actitud de los clínicos docentes hacia las materias, el conflicto entre ambos tipos de profesores, limitación de los recursos y el apoyo financiero, problemas relacionados con el currículum y la actitud negativa de los estudiantes. Estos autores, en su revisión, recogen a su vez las estrategias sugeridas en cada uno de esos dominios para superar las barreras.

Sin embargo, la mayoría de las barreras o problemas identificados e incluidos en este $\mathrm{u}$ otros listados, pero sobre todo las soluciones sugeridas, corren el riesgo de ser vistas como o de difícil implementación o como soluciones simplistas. Usando la metáfora crítica propuesta por Whitehead et al (10) supondrían, a la larga, el retorno regular de los problemas "como los ponis del carrusel dando vueltas repetidas veces en la educación médica". Estos autores (10) realizaron un análisis crítico de los informes educativos, desde los del propio Flexner hasta artículos sobre este tema aparecidos en revistas de educación médica norteamericanas (Academic Medicine y anteriores), e identificaron como los temas que se reivindican son 
La incorporación de las humanidades y ciencias socio-conductuales en la educación médica: ¿cuál es el problema y qué se debe hacer?

Roger Ruiz Moral

recurrentes de tiempo en tiempo a lo largo de todos estos años. Así, estos autores concluyen que existe un discurso el cual, centrándose periódicamente en el futuro, ignora la naturaleza histórica actual de esos problemas. Un discurso que sugiere un sentido de urgencia y propone soluciones que no pasan de ser modestas, en lugar de proponer o implementar los cambios institucionales profundos y sistémicos que serían realmente necesarios (10). De aquí su metáfora de "los ponis del carrusel".

Análisis como los de Bloom (8) o Christakis (9) tienen la ventaja de ofrecer una visión sencilla sobre donde radicaría el problema y, por lo tanto, en teoría, permitirían un abordaje más directo y tal vez eficaz sobre la naturaleza del mismo y sus posibles soluciones. Este artículo quiere insistir en esta línea y llamar la atención sobre la importancia de la lucha ideológica en este terreno y de la hegemonía que con todas las consecuencias está ejerciendo la ideología cientificista biomédica en la educación médica. Consideramos que esta perspectiva puede ser especialmente significativa en este momento, ya que desvela la falacia biomédica de fundamentar sus actuaciones en base a hechos y situaciones de objetiva efectividad (esto es de actuar de forma desideologizada), lo cual representa el núcleo del discurso políticamente correcto de la biomedicina. Muchos de los profesionales y educadores médicos, seguramente de forma inconsciente, defiende en gran medida esta falacia.

De esta manera, quiero resaltar aquí dos componentes principales de la ideología biomédica: por una parte, su carácter pragmatista, el cual descansaría en la consideración de la eficacia del modelo cientificista para conseguir resultados de salud por encima de cualquier otro modelo; y, por otra, su carácter empirista que supone un tipo particular de epistemología y antropología y que apuntaría a un "sentido" de la práctica clínica (y de la labor del médico) eminentemente fisicalista. En el ámbito de la educación médica este modelo enfatiza el desarrollo de una educación que prioriza la adquisición de contenidos y habilidades (competencias) frente al desarrollo de las capacidades metacognitivas del estudiante. 
La incorporación de las humanidades y ciencias socio-conductuales en la educación médica: ¿cuál es el problema y qué se debe hacer?

Roger Ruiz Moral

\section{UNA METÁFORA Y UN MARCO CONCEPTUAL PARA ENTENDER LA FUERZA DE LA IDEOLOGÍA MÉDICA}

\section{LA METÁFORA: LAS MUÑECAS RUSAS}

Desde esta perspectiva, el aspecto nuclear del problema de la integración efectiva de las H\&CC en los curricula de medicina sería por tanto el aspecto ideológico que más arriba señalábamos como preponderante en la educación médica. El cual coloca al modelo biomédico como predominante dentro de ella configurando, por una parte, un currículum siempre insuficiente y siempre sobrecargado, es decir un currículum orientado hacia los contenidos de tipo biomédico y tecnológico y hacia resultados (orientación por competencias), y, por otra, moldeando una mentalidad en sus practicantes que generarían unos hábitos de conducta clínica, educativa y de investigación incompatibles con las H\&CC y resistentes al cambio y a la introducción e integración efectiva de las mismas en la educación médica. Además resulta interesante como este dominio de la biomedicina se extendería más allá del currículum formal ya que los "valores" científicos: competencia, pragmatismo y reduccionismo, marcarían también la mayor parte del currículum oculto imperante en la educación médica (13). Por otra parte, cabe resaltar que este factor "ideológico", recogido en la mayoría de los estudios que abordan el problema, representaría el eje central y generador del resto de los elementos que los diferentes estudios consideran como barreras. Para ello propongo ahora la metáfora de las "muñecas rusas", para enfatizar la inclusión de problemas menores en un problema mayor que los determina (figura 1). Así, la identificación del elemento o elementos claves implicados desvelaría el origen o la principal razón de ser del problema y a la vez de la mayoría de las otras barreras identificadas.

\section{EL MARCO CONCEPTUAL: ARGUMENTOS Y EVIDENCIAS}

Merece la pena aquí ofrecer argumentos y evidencias que faciliten la comprensión del status de la ideología biomédica como ideología dominante en educación médica y sus consecuencias. Con esta finalidad pueden ser útiles los conceptos de "cultura epistémica" de Knorr-Cetina (1999) (14) y el concepto de "campo" acuñado por P Bourdieu (15). El primero es útil porque resalta las 
La incorporación de las humanidades y ciencias socio-conductuales en la educación médica: ¿cuál es el problema y qué se debe hacer?

Roger Ruiz Moral

especificidades de las diferentes culturas científicas y sus prácticas en el ámbito de la salud, en la medida en que nos permite considerar a los diferentes "practicantes de una ciencia" (científicos básicos, clínicos, epidemiólogos, científicos sociales,...) como identificados por diferentes culturas y prácticas. Según Knorr-Cetina, las diferentes culturas epistémicas se revelan mediante distintas aproximaciones empíricas, constructos de significados concretos, "ontologías instrumentales particulares, y maquinarias sociales distintas". La construcción de estas culturas epistémicas está impulsada por los límites institucionales que separan grupos de practicantes o científicos entre sí en organizaciones educativas e investigadoras. Por otra parte, el concepto de "campo" de Bourdieu ayudaría a entender las relaciones de poder entre los grupos, ya que ese concepto es un constructo que se refiere a "relaciones" (p.e., oposición, alianza, dominación, resistencia, etc) entre agentes sociales (individuos, grupos o instituciones) ocupando diferentes posiciones en una red jerárquicamente estructurada. Ambos conceptos se pueden relacionar también con el concepto kuhniano de "paradigma", en el ámbito de la ciencia (16). Así el contexto de la salud puede ser conceptualizado como un campo de producción de conocimiento y de prácticas sobre temas sanitarios en el que diferentes grupos interactúan en consonancia con su propia cultura epistémica. La cultura epistémica hegemónica es aquí la biomédica. En términos kuhnianos de la biomedicina ocuparía el "paradigma dominante" en el ámbito científico de las ciencias de la salud (16). Así, las relaciones de poder en el ámbito de la salud y de la educación médica, como en todos los campos, gira en torno a lo biomédico y su visión biológica de la salud: enfermedad como alteración anatómica o bioquímica con un paradigma investigador cuantitativo experimental, la cual se constituye como ciencia legítima. Usando ambos conceptos, cultura y campo epistémico, podemos entender mejor cómo esta cultura epistémica imperante y hegemónica en la práctica, la investigación y la educación médica (paradigma dominante) funciona como árbitro del tipo de conocimiento que tiene o no valor y de la producción que generan otras culturas epistémicas. De esta forma el valor de la cultura epistémica y las prácticas de investigación de los científicos sociales, con un concepto de la salud más holístico, con enfoques investigadores más cualitativos, recién llegados a la medicina o con una posición tradicionalmente secundaria, estará determinado en 
La incorporación de las humanidades y ciencias socio-conductuales en la educación médica: ¿cuál es el problema y qué se debe hacer?

Roger Ruiz Moral

gran medida por las percepciones y juicios de los clínicos, educadores y científicos biomédicos dotados con el poder de definir lo que es valioso en el campo de la medicina y lo que no. A este propósito, el uso del concepto de campo de Bourdieu facilita percatarse de la existencia de una lucha de poder donde los científicos biomédicos y su cultura epistémica ocupa una posición dominante para defender las políticas sanitarias, educativas y de investigación y el acceso a recursos en cualquiera de estos tres ámbitos frente a otros "competidores" con culturas epistémicas diferentes.

Podemos encontrar constancia de la existencia de esta dinámica en el trabajo de Albert et al (17). Estos autores exploran las posibilidades que tienen las ciencias sociales para integrarse y prosperar en un campo en el que el paradigma experimental lo ocupan la ciencia biomédica. Mediante encuestas en profundidad realizadas a 31 científicos biomédicos, estos autores constataron como la gran mayoría de ellos (16 científicos) sostuvieron que las ciencias sociales no podían generar resultados válidos y fiables porque no asumen el diseño experimental como un enfoque metodológico en sus investigaciones, mientras otros ( 7 científicos) mostraron una postura de aceptación cautelosa hacia las ciencias sociales especialmente por sus reservas sobre los métodos cualitativos de investigación. De esta manera estos autores concluían que la incorporación de las ciencias sociales continuará encontrando obstáculos en el campo de la investigación de la salud en el futuro. Litva y Peters (18) tras realizar una encuesta a educadores médicos sobre los problemas para introducir las H\&CSC, utilizan el descriptivo y belicoso término de "epistemologías atrincheradas" para describir las resistencias belicosas que describen los encuestados, en el ámbito educativo médico, donde la hegemonía biomédica se reflejaría tanto en los métodos evaluativos utilizados como en los criterios que las escuelas de medicina utilizan para aceptar a los estudiantes. El predominio de determinadas técnicas evaluativas en las escuelas de medicina, como por ejemplo las preguntas de elección múltiple o los formatos de examen tipo ECOE que valoran un conocimiento de tipo factual excluye otros tipos de conocimiento. Son varios los autores que han llamado la atención sobre como esos métodos evaluativos tradicionales utilizados en las escuelas de medicina suponen una barrera para el afianzamiento de la enseñanza de H\&CC $(11,19)$. Esta diferencia de status y 
La incorporación de las humanidades y ciencias socio-conductuales en la educación médica: ¿cuál es el problema y qué se debe hacer?

Roger Ruiz Moral

de poder entre medicina y humanidades también queda patente en el estudio de Wachter et al (20). En este estudio, al explorar los problemas que aparecen cuando se incorpora un currículum de tipo interdisciplinar en humanidades médicas, las autoras observan como esas diferencias en el status representan una barrera mayor para una implementación exitosa del Programa de Humanidades Médicas. Aunque el programa de humanidades tenía una intención de igualdad en su ideología, "ya desde el principio esto se contradecía por el enfoque que se hizo del programa hacia la necesidad del estudiante de medicina, y por la relación de poder existente entre las facultades de medicina y de humanidades que esto reflejaba”. Así, en la práctica los contenidos de las "humanidades" eran construidos, definidos y utilizados dentro de un marco de referencia "médico", donde las "humanidades" se supeditaban a los médicos y se construían como materias "opuestas" e inferiores: una especie de "nociencia", "no-práctica", "no académica", complementaria y no igual a la medicina, predominando una aproximación racionalista al conocimiento que no facilitaba la estructura teórica suficiente a los estudiantes como para entender en profundidad lo que aprendían. Para las autoras, este diferencial de poder hizo imposible la construcción de un ambiente verdaderamente interdisciplinario, donde una representación e interacción igualitaria de las diferentes disciplinas pudiese dar lugar a una "perspectiva metodológica más amplia" en la que aparecieran nuevas formas de investigar y resolver los problemas (20).

Los estudiantes no son inmunes a este dominio epistemológico y aunque estos parecen expresar niveles predominantemente simplistas de pensamiento epistemológico (las creencias personales sobre qué es el conocimiento y cómo lo entendemos, integramos y aplicamos) en el que influyen el conocimiento científico y el experiencial, un entrenamiento médico dominado por el pensamiento científico viene finalmente a inclinar la balanza hacia identidades profesionales donde predomina el pensamiento biomédico y a su vez el rechazo de las H\&CSC (21).

\section{DOS ESTRATEGÍAS PARA INTRODUCIR LAS H\&CSC EN MEDICINA}

Antes destacábamos dos de las características por las que el modelo biomédico ha afianzado su hegemonía en la educación médica: su ideología empirista y reduccionista, y su enfoque eminentemente práctico o utilitarista. Pues 
La incorporación de las humanidades y ciencias socio-conductuales en la educación médica: ¿cuál es el problema y qué se debe hacer?

Roger Ruiz Moral

bien, las alternativas que propongo aquí deberían priorizarse para acabar con esta hegemonía siguen estos dos mismos frentes. Por una parte, esta filosofía en la que se basa la biomedicina siendo parcial y ofreciendo una visión del mundo muy reducida y hoy día insuficiente para comprender muchos de los problemas en nuestro caso de la práctica clínica (carácter crónico de las enfermedades, multimorbididad, dinámica de la toma de decisiones médicas, papel preponderante de la incertidumbre tanto en la causalidad como en la solución de los problemas de salud, caracterización de las influencias sociales en los procesos de saludenfermedad, la propia naturaleza del razonamiento clínico, etc), debería ser reemplazada por una visión más amplia que incluya perspectivas epistemológicas y antropológicas más satisfactorias y explicativas. En diferentes foros universitarios, intelectuales y religiosos se ha denominado a esta necesidad como la necesidad de repensar los supuestos epistemológicos y antropológicos en los que basamos el saber de nuestro campo particular para "ampliar la razón" (22). La característica básica de esta perspectiva es que con ella se reivindica desde el mundo educativo en general y universitario en particular una visión más ampliada del mundo y por tanto un mayor y más certero acercamiento en la búsqueda de "la verdad" (23), como tarea propia de la empresa educativa desde los orígenes de nuestra cultura (24). La otra línea de actuación se centra en el carácter utilitarista que viene utilizando la biomedicina, reivindicando así una eficacia de sus metodologías superior a las utilizadas por otras ramas del saber, en especial por las humanidades y ciencias sociales. Pues bien, las H\&CSC deben también de hacer valer su valor pragmático para resolver algunos de los problemas más importantes que actualmente tiene planteados la clínica. Estas dos perspectivas no pretenden anular o imponerse a la visión y métodos biomédicos sino ampliar el campo de acción para comprender mejor los problemas y su eficacia para resolverlos, desde una condición de igualdad.

\section{PROMOVER UN CAMBIO DE MENTALIDAD EN LA EDUCACIÓN: LA "RAZÓN ABIERTA"}

El marco general de la educación médica es el marco de la enseñanza, por tanto, la pregunta principal que debería de responderse aquí es: “¿para qué 
La incorporación de las humanidades y ciencias socio-conductuales en la educación médica: ¿cuál es el problema y qué se debe hacer?

Roger Ruiz Moral

enseñamos?" La respuesta a esta pregunta debería de ser integral y abarcadora, así, escogemos la siguiente definición de Lacalle (22) "sobre todo, para la vida, para que sepan desentrañar la realidad y comprender el mundo, para que asuman con responsabilidad su proyecto de vida, para que se desarrollen plenamente como personas y sirvan al bien común". Especificamos para el caso que nos ocupa, la educación médica, donde "desentrañar la realidad y comprender el mundo" tiene que ver específicamente con desentrañar la realidad de la dolencia y promover la salud, entendida esta en el sentido holístico del término. Y donde, "servir al bien común" es resolver los problemas de salud de los pacientes y la comunidad. Sin embargo lo anterior implica el interiorizar que el proyecto educativo supone, también en medicina, una necesidad de ampliar la mentalidad del estudiante y del médico. El pensador cristiano y papa Benedicto XVI lo ha expresado con mucha claridad: "La intención no es retroceder o hacer una crítica negativa, sino ampliar nuestro concepto de razón y de su uso" (25) y "La propuesta de ensanchar los horizontes de la racionalidad no debe incluirse simplemente entre las nuevas líneas de pensamiento teológico y filosófico, sino que debe entenderse como la petición de una nueva apertura a la realidad a la que está llamada la persona humana en su unitotalidad, superando antiguos prejuicios y reduccionismos, para abrirse también así el camino a una verdadera comprensión de la modernidad" (26).

Sin embargo y contra lo que se puede pensar, esta perspectiva ha estado presente desde el inicio de la moderna educación médica, ya que identificamos este mismo mensaje en la perspectiva del propio Abraham Flexner, el cual en sus dos informes de 1910 y 1925, tradicionalmente se considera que origina el nacimiento y estructuración de la moderna educación médica. Whitehead ha realizado un análisis foucaultdiano de esos dos textos en lo que respecta al predominio del discurso de las ciencias biomédicas frente a las ciencias humanas (27). El análisis que esta autora hace de sendos informes revela la noción cambiante de lo que para Flexner es un "buen médico" (good doctor) (que él lo cataloga en función del conocimiento, identidad y responsabilidad social). Flexner liga la noción de médico-científico (scientist-doctor) a aquél que posee un pensamiento incisivo, con un conocimiento obtenido de múltiples fuentes, incluyendo las ciencias naturales, las sociales y las humanidades. El concepto de "ciencia" para Flexner no es el de una mera 
La incorporación de las humanidades y ciencias socio-conductuales en la educación médica: ¿cuál es el problema y qué se debe hacer?

Roger Ruiz Moral

herramienta de la que se deben de aprovechar los médicos, sino que una aproximación "científica" es más bien una forma de ser. Él hizo un énfasis especial entre la necesidad de no separar el espíritu de la investigación y el de la práctica, siendo éste el aspecto clave que hace a un médico también ser un científico. En su discurso, señala Whitehead, una aproximación de búsqueda (inquiring approach) es el elemento clave, así para Flexner "Ciencia" es esencialmente un "estado mental": "El buscador científico reúne hechos de las fuentes disponibles mediante cualquier medio posible. La Ciencia reside en el intelecto, no en los instrumentos" (28). Flexner rechaza empáticamente una noción de ciencia restringida, es decir una ciencia "estrictamente confinada a un conocimiento capaz de expresarse cuantitativamente y para ser utilizado". El asumir esta perspectiva exige incluir a las ciencias sociales y a las humanidades, y enfatiza la necesidad de adoptar una actitud intelectual para el enfoque científico basado en "el persistente esfuerzo del hombre para purificar, ampliar y organizar su conocimiento del mundo" (28).

Por todo lo anteriormente expuesto, creo que la consideración que Flexner tenía sobre el concepto de "ciencia" era el de una "razón abierta o ampliada" (Expanded Reason) y su enfoque sobre la educación médica, como aquél que prioriza el desarrollo intelectual del estudiante de medicina por encima de los detalles de un contenido curricular de un tipo $u$ otro.

El análisis discursivo de los informes de Flexner, hecho por Whitehead también resalta que su visión del médico-científico permite la inclusión de distintas áreas de aprendizaje, y todas ellas pueden y deben de ser objeto de utilización rigurosa por parte de este médico-científico: “...El clínico trata con hechos de dos categorías. La química, física, biología, le capacitan para aprehender las de un tipo; precisando un aparato apreciativo diferente para tratar las de otro tipo, elementos más sutiles. Una preparación específica para esto es mucho más difícil; uno debe confiar en el requisito de la introspección (insight) y la simpatía, basándose en una variada y amplia experiencia cultural" (29).

Fue después de las reformas de Flexner cuando la noción de "ciencia biomédica" se hizo dominante en el conocimiento médico y él mismo manifestó su disgusto por este hecho, así en el informe de 1925 se lamentaba de esta forma: "La 
La incorporación de las humanidades y ciencias socio-conductuales en la educación médica: ¿cuál es el problema y qué se debe hacer?

Roger Ruiz Moral

medicina científica en América -joven, vigorosa, positivista- es hoy tristemente deficiente en su base cultural y filosófica" (28)

Whitehead amplía su análisis a los textos que sobre el tema se publicaron en las revistas académicas americanas de la época y posteriormente en Academic Medicine (hasta el 2010) (27). Su estudio ofrece una interesante perspectiva para comprender la preponderancia posterior adquirida por la ideología biomédica, cual es la percepción de la "ciencia" como un objeto discursivo inmerso en el curriculum por encima de la persona del "científico" como "sujeto discursivo" que utiliza la ciencia. De esta manera la "medicina científica" habría reforzado la centralidad de la biomedicina en la educación médica, contribuyendo así a la marginalización de otros dominios de conocimiento importantes como los humanísticos y los de las ciencias sociales.

\section{PROMOVER UN ENFOQUE TAMBIÉN UTILITARISTA DE LAS H\&CSC EN LA PRÁCTICA MÉDICA}

El fundamento de la otra línea de actuación que propongo, se encuentra en el hecho de que la incorporación de las humanidades en medicina se ha visto durante bastantes años envuelta en la estéril discusión sobre la necesidad real de las razones por las que aquellas debían incorporarse a los currícula médicos. Esta necesidad trataba, también estérilmente, de fundamentarse o bien en una utilidad de las humanidades para el ejercicio efectivo de la práctica o simplemente como algo que tiene "valor por sí mismo", es decir por una especie de necesidad de apreciar una dimensión puramente "estética", sin que esto suponga a medio o largo plazo ningún cambio en las capacidades del perceptor de tal formación (30). En palabras de S Álvarez, algo así como el "spa" del médico, un lugar para el relax y disfrute personal, pero sin repercusión en su labor profesional (31). En esta dirección abunda J Macnaughton, una de las teóricas más reputadas en humanidades médicas, la cual habla del valor "no instrumental" de las humanidades en base a su valor educativo (entendido éste como una perspectiva más amplia que la puramente "de entrenamiento"). Algo así cómo para el desarrollo de la persona y, a la vez, como herramienta para contrapesar la cultura imperante en medicina (30). Charlotte Blease ha resaltado como esta perspectiva del "conflicto" (instrumental vs no 
La incorporación de las humanidades y ciencias socio-conductuales en la educación médica: ¿cuál es el problema y qué se debe hacer?

Roger Ruiz Moral

instrumental) no es más que una falsa dicotomía que se inserta en una estéril discusión similar a la de la educación vs el entrenamiento o lo teórico vs lo práctico (32). Esta autora ve en esta falsa dicotomía sin embargo una contribución que ahonda la brecha existente entre las humanidades y las ciencias a la vez que oculta la potencial utilidad de las humanidades para los médicos (32).

El carácter utilitarista que viene utilizando la biomedicina reivindica una eficacia de sus metodologías superior a las utilizadas por otras ramas del saber, en especial por las humanidades y ciencias sociales. Opino que frente a esto y como complemento a la estrategia anterior, las H\&CSC deben de reivindicar su valor pragmático para resolver algunos de los problemas más importantes que actualmente se plantea la clínica. Las H\&CSC contribuyen y, más aún en el momento actual, son indispensables para el desarrollo de las capacidades clínicas de los médicos y su incorporación a los currícula debería resaltar también esta perspectiva utilitarista y no la de pretender aportar exclusivamente un valor educativo y/o de desarrollo personal o la de ofrecer una visión negativa cual es la de servir de mero contrapeso o contenedor de la biomedicina (31). Alineándome con las tesis de partida de Blease esta falta de visión utilitarista contribuye al afianzamiento de la ideología que ha dominado tanto la práctica clínica como la educación médica desde el establecimiento de los modernos sistemas educativos médicos, condicionando los hábitos en el mundo académico de la medicina y dando origen a algunas de las principales barreras para la incorporación e integración de las humanidades en los currícula médicos.

Baste decir aquí ahora que la utilidad general de las humanidades médicas (y me refiero tanto a las humanidades propiamente dichas: literatura y narrativa, arte, filosofía, historia, como a las ciencias sociales y conductuales: sociología, economía de la salud, salud pública, antropología, psicología y relaciones interpersonales) (H\&CC) en la educación médica de forma genérica contribuyen a ofrecer a los estudiantes de medicina y a los médicos una formación que les permitiría explorar la "vida" de los pacientes en todas sus dimensiones y su impacto en la enfermedad y el de esta en la propia vida. Estas materias promueven el desarrollo en los médicos de la sensibilidad necesaria para afrontar y comprender con sensibilidad la amplia gama de experiencias de enfermedad de sus pacientes y los dilemas morales a los que 
La incorporación de las humanidades y ciencias socio-conductuales en la educación médica: ¿cuál es el problema y qué se debe hacer?

Roger Ruiz Moral

diariamente se enfrentan, pero también ofrecen herramientas prácticas para hacer de la intervención clínica una intervención más adecuada y eficaz. Desde puntos de vista más específicos, las diferentes materias humanísticas y conductuales incluidas en los currícula médicos contribuirían, entre otros aspectos a "promover una atención clínica centrada en el paciente" (33); "facilitar el desarrollo de empatía y afectividad en los médicos (34); "ayudar a desarrollar una práctica clínica prudente y realista (phronesis) refinando y sopesando sus juicios" (35); "evitar o disminuir el sufrimiento personal que conlleva el ejercicio de la práctica (burnout) $(33,36)$; " contribuir al desarrollo de actitudes médicas más humildes" (32); "facilitar la investigación interdisciplinaria" (33); "fomentar una actitud crítica e inquisitiva ante los problemas y ante las evidencias científicas" (36,37); "desarrollar la moral profesional" (31,33); "contribuir al crecimiento personal de los estudiantes de medicina y los médicos" (39) "aumentar la comprensión de la condición humana" (38); "mejorar la interacción con los pacientes" (38), o "conseguir mejores resultados de salud" (40). Como puede verse, en la medicina y su práctica la amplitud de campos en los que las H\&CSC pueden contribuir para mejorar la labor social del clínico es muy amplia, y sería responsabilidad de éstas desarrollar una agenda de investigación lo suficientemente potente como para poner de manifiesto esta utilidad.

\author{
Roger Ruiz Moral \\ Profesor de Medicina. \\ Universidad Francisco de Vitoria (Madrid) \\ rruizm@semfyc.es
}

\title{
REFERENCIAS
}

1. Finnerty EP, Chauvin S, Bonaminio G, Andrews M, Carroll RG, Pangaro LN. Flexner revisited: the role and Blackwell Publishing Ltd 2013. Discourses of science in medical education value of the basic sciences in medical education. Acad Med 2010;85:349-55.

2. Weatherall D. Science and medical education: is it time to revisit Flexner? Med Educ 2011;45:4450 .

3. Kuper A, D'Eon M. Rethinking the basis of medical knowledge. Med Educ 2011;45:36-43.

4. Whitcomb ME. The general professional education of the physician. Acad Med 2006;81:1015-6.

5. Sales CS, Schlaff AL. Reforming medical education: a review and synthesis of five critiques of medical practice. Soc Sci Med 2010;70:1665-8.

6. Whitehead C. Recipes for medical education reform: will different ingredients create better doctors? A commentary on Sales and Schlaff. Soc Sci Med 2010;70:1672-6. 
La incorporación de las humanidades y ciencias socio-conductuales en la educación médica: ¿cuál es el problema y qué se debe hacer?

7. Smyth FS. The place of the humanities and social sciences in the education of physicians. J Med Educ 1962;37:495-9.

8. Bloom SW. Structure and ideology in medical education: an analysis of resistance to change. $\mathrm{J}$ Health Soc Behav 1988;29:294-306

9. Christakis, N. A. The similarity and frequency of proposals to reform US medical education. Journal. of the American Medical Association, 1995;274(9):706-711

10. Whitehead C, Hodges BD, Austin Z. Captive on a carousel: discourses of "new" in medical education 1910-2010. Adv in Health Sci Educ 2013;18:755-768

11. Russell $A$, van Teijlingen E, Lambert $H$, Stacy R. Social and behavioural science education in UK medical schools: current practice and future directions. Med Educ 2004;38:409-17.

12. Tabatabaei, Z., Yazdani, S., Sadeghi, R. (2016). Barriers to integration of behavioral and social sciences in the general medicine curriculum and recommended strategies to overcome: $\mathrm{A}$ systematic review. J Adv Med Educ Prof, 4, 111-21

13. Lempp $\mathrm{H}$, Seale $\mathrm{C}$. The hidden curriculum in undergraduate medical education: qualitative study of medical students_perceptions of teaching. BMJ 2006;329:770-3.

14. Knorr-Cetina, K. Epistemic cultures. How the sciences make knowledge. Cambridge, MA: Harvard University Press, 1999

15. P Bourdieu. El oficio de científico: Ciencia de la ciencia y reflexividad. Barcelona: Anagrama, 2003

16. Kuhnn TS. La estructura de las revoluciones científicas. Madrid: FCE de España, 2011.

17. Albert M, Laberge S, Hodges B, Regehr G, Lingard L. Biomedical scientists' perception of the social sciences in health research. Soc Sci Med 2008;66:2520-31

18. Litva A, Peters S. Exploring barriers to teaching behavioural and social sciences in medical education. Medical Education 2008;42:309-314

19. Satterfield JM, Mitteness LS, Tervalon M, Adler N. Integrating the social and behavioural sciences in an undergraduate medical curriculum: the UCSF essential core. Acad Med 2004;79:6-15

20. Wachtler C, Lundin S, Troein M. Humanities for medical students? A qualitative study of a medical humanities curriculum in a medical school program. BMC Medical Education 2006;6:16

21. Knight LV, Mattick K. When I first came here, I thought medicine was black and white: making sense of medical students ways of knowing. Soc Sci Med 2006;63:1084-96

22. Lacalle M. En busca de la unidad del saber. Una propuesta para renovar las disciplinas universitarias. Editorial UFV, 2018 (segunda edición)

23. Misión UFV. Nuestra Misión hoy. Madrid: Universidad Francisco de Vitoria, 2010, pp. 43-45

24. Jaeger W. Paideia: Los ideales de la cultura griega. Madrid: Fondo de Cultura Económica de España, SL, 2007

25. Benedicto XVI, Fe, razón y universidad, Discurso pronunciado en la universidad de Ratisbona, 12 de septiembre de 2206.

26. Benedicto XVI Discurso inaugural del VI Simposio europeo de profesores universitarios sobre el tema: Ensanchar los horizontes de la racionalidad. Perspectivas para la filosofía. 27 de junio del 2008

27. Whitehead C. Scientist or science-stuffed? Discourses in North America medical education. Med Edu 2013;47:26-32

28. Flexner A. Medical Education: A Comparative Study. New York, NY: Macmillan Company 1925

29. Flexner A. Medical Education in the United States and Canada: A Report to the Carnegie Foundation for the Advancement of Teaching. New York, NY: Arno Press 1972 (reprint).

30. Alvarez Montero S. Las Humanidades médicas: el spa del clínico. Comunicación personal al autor, 2017

31. Macnaughton J. The humanities in medical education: context, outcomes and structures. Med Humanit 2000;26:23-30

32. Blease C. In defence of utility: the medical humanities and medical education. Med Humanit 2016;42:103-8

33. Gordon J. Medical humanities: to cure sometimes, to relieve often, to comfort always. Med J Aust 2005;182:5-8.

34. Jackson M. Medical humanities in medical education. Med Educ 1996;20:395-6.

35. Shapiro J, Coulehan J, Wear D, et al. Medical humanities and their discontents: definitions, critiques, and implications. Acad Med 2009;84:192-8.

36. Wilcock SM, Daly MG, Tennant CC, et al. Burnout and psychiatric morbidity in new medical graduates. Med J Aust 2004;181:357-60. 
La incorporación de las humanidades y ciencias socio-conductuales en la educación médica: ¿cuál es el problema y qué se debe hacer?

Roger Ruiz Moral

37. Downie R, Hendry RA, Macnaughton RJ, et al. Humanizing medicine: a special study module. Med Educ 1997;31:276-80.

38. Grant VJ. Making room for medical humanities. Med Humanit 2002;28:45-8

39. Ruiz Moral R. Propuesta educativa en comunicación clínica y su influencia en la reflexión de los alumnos de medicina sobre aspectos epistemológicos, antropológicos, éticos y de sentido. II Congreso Razón Abierta: Roma 26 y 27 de Septiembre 2018

40. Levinson W, Lesser CS, Epstein RM. Developing Physician Communication Skills For PatientCentered Care. Health Affaires 2010;29:1310-8

Figura 1. La metáfora de las Muñecas Rusas: influencia de la hegemonía biomédica (epistemología atrincherada) sobre las barreras para la incorporación de las H\&CSC en los currículos médicos

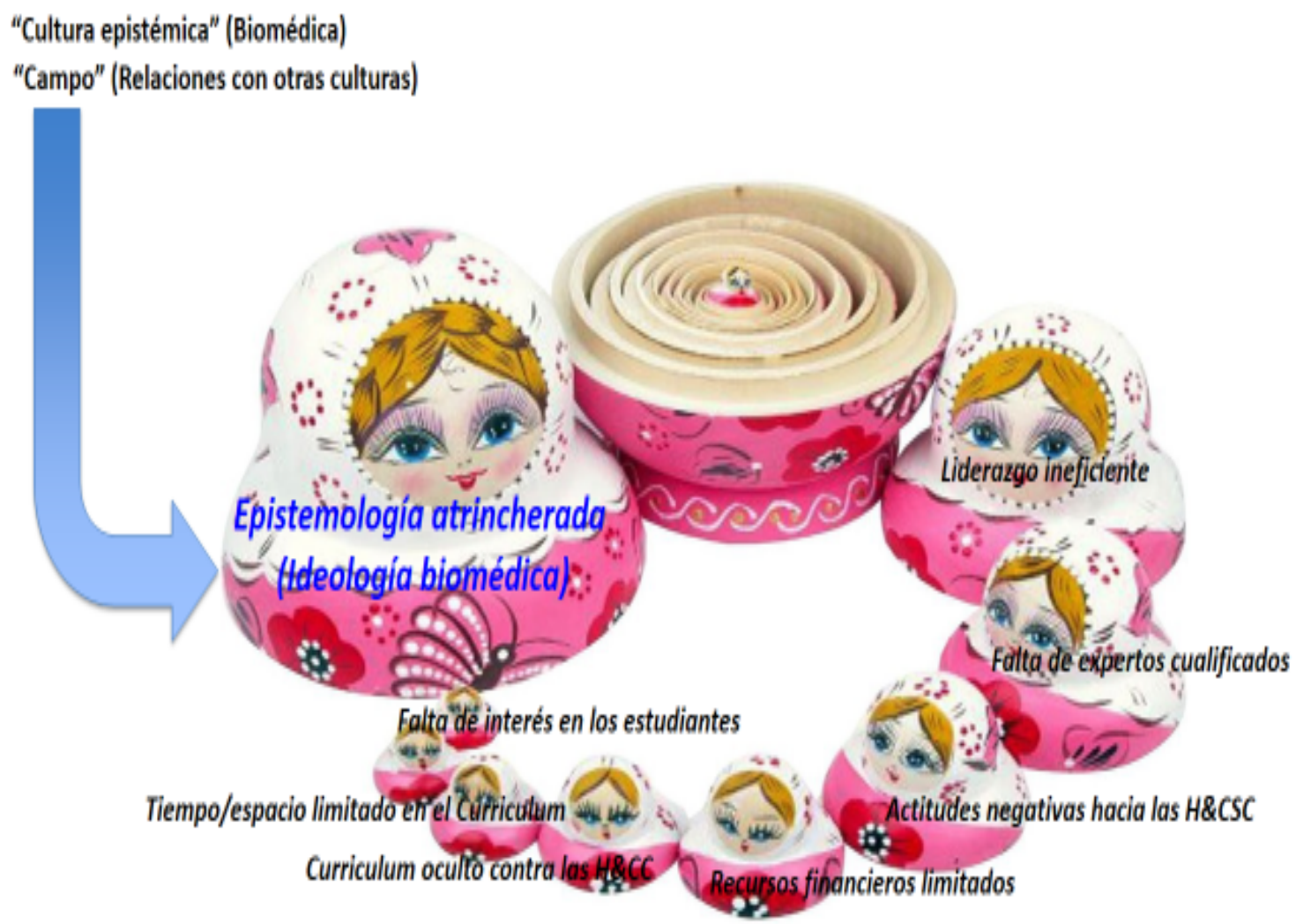

\section{Cómo citar este artículo:}

Ruiz Moral, R., "La incorporación de las Humanidades y Ciencias Socio-Conductuales en la educación médica", Folia Humanística, 2019 (11): 65- 81. Doi:

http://dox.doi.org/10.30860/0050.

(C) 2019 Todos los derechos reservados a la Revista Folia Humanística de la Fundación Letamendi Forns. This is an open access article. 